valve endocarditis involving the mitral annulus. Ann Thorac Surg. 2009;88: e29-30.

6. David TE. The use of pericardium in acquired heart disease: a review article. J Heart Valve Dis. 1998;7:13-8

7. Acar C, Saez de Ibarra J, Lansac E. Anterior leaflet augmentation with autologous pericardium for mitral repair in rheumatic mitral insufficiency. J Heart Valve Dis. 2004; 13:741-6.
8. Chauvaud S, Jebara V, Chachques J-C, El Asmar B, Mihaileanu S, Perier P, et al Valve extension with glutaraldehyde-preserved autologous pericardium: results in mitral valve repair. J Thorac Cardiovasc Surg. 1991;102:171-8.

9. Acar C, Tolan M, Berrebi A, Gaer J, Gouezo R, Marchix T, et al. Homograft replacement of the mitral valve: graft selection, technique of implantation, and results in forty-three patients. J Thorac Cardiovasc Surg. 1996;111: 367-80.

\title{
Open antegrade aortic stent implantation after surgical treatment in type A aortic dissection
}

\author{
Franz Masseli, MD, ${ }^{\mathrm{a}}$ Kai Wilhelm, MD, PhD,${ }^{\mathrm{b}}$ Chris Probst, MD, ${ }^{\mathrm{a}}$ and Wolfgang Schiller, MD, ${ }^{\mathrm{a}}$ Bonn, \\ Germany
}

Major complications after surgery for Stanford type A aortic dissections are aortic rupture and malperfusion of abdominal organs. The choice between subsequent surgical replacement of the descending aorta and retrograde stent placement via the femoral artery depends on the size of the true lumen and the location, as well as the extent of the intimal tear. Because the stent graft has to be advanced through segments with decreased wall strength, aortic rupture may be caused during the passage or the stent may be released in the false lumen. ${ }^{1}$ To minimize these risks, interventional techniques using an antegrade approach via the subclavian artery or the use of a hybrid procedure with open implantation of an aortic stent before placement of the arch prosthesis have recently been described. ${ }^{2-4} \mathrm{We}$ developed a new and safe technique of antegrade stent graft placement via the already implanted prosthesis either after resternotomy or during the original procedure. This technique aims to improve patient survival while minimizing postoperative complications because the stent is inserted through the firmest aortic segment, the prosthesis, in an antegrade fashion.

\section{MATERIALS AND METHODS}

The stent graft placement depends on the extent of the previously implanted aortic prosthesis and, in cases of concomitant arch replacement, on the technique used for the connection of the supraaortic vessels to the prosthesis (Figure 1). The preoperative imaging method of choice for this evaluation is an electrocardiogram-gated, multislice computed tomography (CT) examination of the entire aorta and supra-aortic branches.

From the Departments of Cardiac Surgery ${ }^{\mathrm{a}}$ and Radiology, ${ }^{\mathrm{b}}$ University Hospital Bonn, Bonn, Germany.

Disclosures: Authors have nothing to disclose with regard to commercial support. Received for publication May 7, 2012; revisions received June 30, 2012; accepted for publication July 30, 2012; available ahead of print Aug 30, 2012.

Address for reprints: Franz Masseli, MD, Sigmund-Freud St 25, 53127 Bonn,

Germany (E-mail: fmasseli@uni-bonn.de).

J Thorac Cardiovasc Surg 2012;144:1527-30

$0022-5223 / \$ 36.00$

Copyright $\odot 2012$ by The American Association for Thoracic Surgery

http://dx.doi.org/10.1016/j.jtcvs.2012.07.088
Before the procedure, written consent was obtained from the patient or the next of kin. The procedure was carried out on a Compassionate Use basis according to German law.

After sternotomy, surgical clips at the aortic wall or the implanted prosthesis can be used to mark the proximal landing zone of the stent. To allow an unimpeded delivery of the stent past the end of the introducer, the point of introduction (POI) is chosen at least $3 \mathrm{~cm}$ proximal to the desired landing zone. A purse-string suture is then placed around the POI at the wall of the prosthesis.

Heparin is administered, followed by the antegrade introduction of an angio/pigtail catheter into the true aortic lumen via a 7F introducer sheath at the selected POI. The catheter is advanced into the aortic arch, and digital subtraction angiography is performed during breathhold to outline the aorta and supra-aortic branches and to identify the placed markers. The catheter is then exchanged for a flexible guidewire (Radifocus; Terumo Medical Corp, Eschborn, Germany). The introduction into the true lumen is verified using transesophageal echocardiography visualization. The flexible guidewire is then exchanged for a stiff guidewire (Lunderquist extra-stiff; Cook Medical Inc, Bloomington, Ind). A stent graft with a suitable design for antegrade delivery is selected, and its introducer sheet is marked approximately $3 \mathrm{~cm}$ away from its end, depending on the distance of the POI to the planned proximal landing zone. After removing the catheter and the $7 F$ sheath, the introducer is advanced over the guidewire up to the $3-\mathrm{cm}$ mark. A small incision while passing the wall of the prosthesis is performed to facilitate the insertion. Under radiographic control, the stent is advanced via the introducer. In cases in which the POI is located proximal to the arch, the stent graft is manually bent before insertion to facilitate an easy passage through the aortic arch. The insertion is continued up to the desired position, which is verified by fluoroscopy via its projection in relation to the radio-opaque clips. The stent is then released, and the introducer system is removed.

During the release of the stent graft, blood pressure is decreased to less than $100 \mathrm{~mm} \mathrm{Hg}$ systolic; because of the fast-expanding capacity, no further modulation of cardiac output, such as rapid pacing, is necessary. The proximal aspect of the stent can be secured in the region of the overlapping landing zone with 2 Teflon-armed transmural sutures to prevent dislocation of the device. After radiographic control of accurate stent placement, the guidewire and introducer sheet are removed while simultaneously tightening the purse-string sutures. In cases with prior arch replacement, the proximal landing zone should be within the distal portion of the implanted prosthesis.

\section{RESULTS}

To the present, we have used this technique in a series of 5 patients with type A aortic dissection. None of the patients 

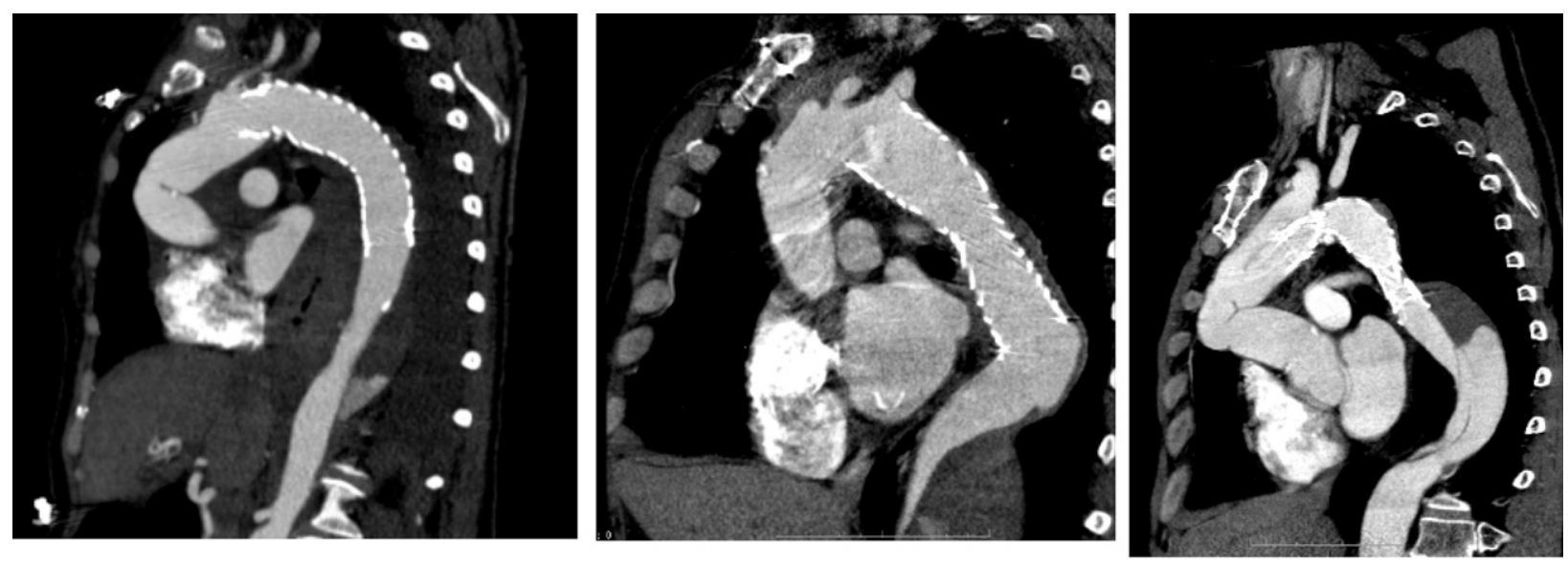

A
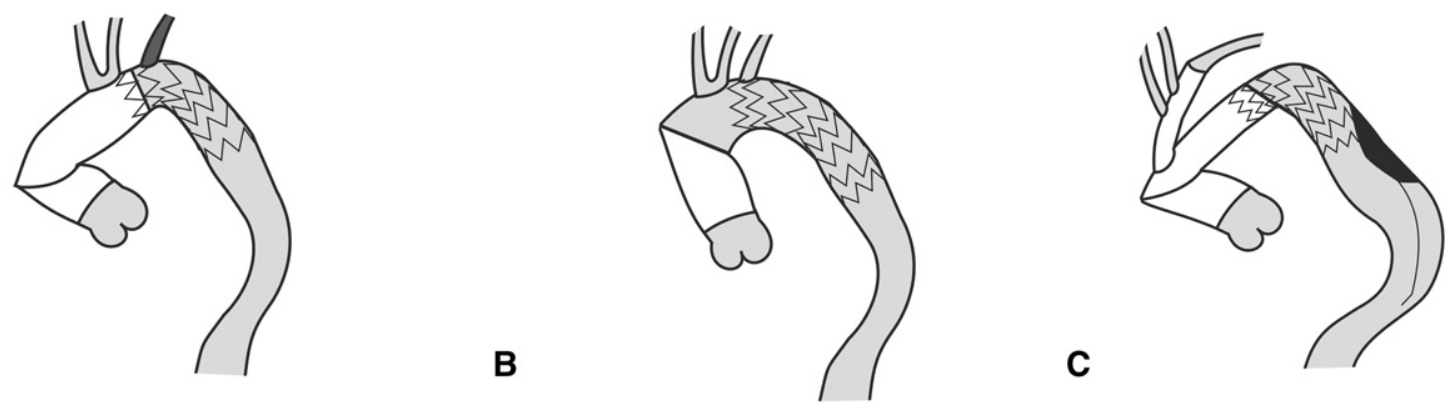

FIGURE 1. CT scan on postoperative day 8: Primary aortic replacement up to the LSA is shown; the stent is overlapping the prosthesis and covering the LSA (A). Postoperative day 3: Primary replacement of the ascending aorta and remaining perfusion of the LSA after placement of the stent graft are shown (B). Postoperative day 6: Complete arch replacement is shown; the stent is overlapping the distal end of the prosthesis without blocking perfusion of the supracoronary vessels; in the distal portion of the stent, thrombosis of the false lumen can be seen (C). LSA, Left subclavian artery.

showed signs of connective tissue disorder or had a family history of aortic disease.

In all of the treated patients, the technique was technically and clinically successful. Of these 5 patients, 4 received treatment in a separate operation because of delayed onset of complications. New neurologic complications, wound infection, or mediastinitis did not develop in any of the patients. In 1 patient, antegrade implantation was performed during the primary operation (total arch replacement); the stent was successfully implanted to treat uncontrollable bleeding by covering the distal anastomosis site after arch replacement.

We present 3 exemplary cases, each with a different aortic anatomy:

\section{Case 1: Redissection and Rupture}

A 65-year-old woman with type A dissection extending to the left subclavian artery (LSA) received a replacement of the dissected ascending aorta using a 28-mm Hemashield platinum prosthesis (Maquet $\mathrm{GmbH}$, Rastatt, Germany). The distal anastomosis was performed just proximal to the LSA; the brachiocephalic trunk and left carotid were reimplanted in an insular technique.

After an initially fast postoperative recovery, the patient experienced severe thoracic-lumbar pain 10 days later.
A CT scan revealed an extensive redissection of the descending aorta starting from the distal anastomosis site. A large para-aortic hematoma severely compressed the left atrium, leading to flow acceleration and a new onset of dyspnea. Para-aortic contrast agent in a CT scan supported the diagnosis of aortic perforation. After resternotomy, an endovascular stent (Gore TAG, $34 \mathrm{~mm} \times 15 \mathrm{~cm}$; WL Gore \& Associates, Inc, Flagstaff, Ariz) was implanted using the technique described earlier. The stent covered $2 \mathrm{~cm}$ of the aortic prosthesis, the LSA, and the perforated aortic segment. The patient had a transient paraplegia postoperatively, which resolved completely before discharge 5 weeks later. CT follow-up showed a progressively thrombosing false lumen and a regression of the para-aortic hematoma (Figure 1, A).

\section{Case 2: Abdominal Malperfusion Syndrome}

A 53-year-old man with type A dissection of the whole aorta received a supracoronary replacement of the ascending aorta and the proximal arch (Hemashield Platinum, $28 \mathrm{~mm}$ ). The patient was paraplegic postoperatively. He had abdominal pain and an ongoing lactate acidosis. CT scan showed an almost complete occlusion of the true lumen at the level of the descending thoracic aorta. An endovascular stent (Gore TAG, $36 \mathrm{~mm} \times 15 \mathrm{~cm}$ ) was implanted immediate distally to the LSA in an antegrade 
technique. When addressing the limited extent of the primary implanted vascular prosthesis, the POI was selected at the lateral aspect of the prosthesis. The sheath and stent were manually bent before insertion through the aortic arch. After stent implantation, lactate levels decreased and abdominal symptoms improved while the paraplegia remained (Figure 1, B).

\section{Case 3: Acute Liver Failure Due to Malperfusion}

A 69-year-old man with acute type A dissection received supracoronary aortic replacement, including the complete aortic arch because of the extent of intimal disruption. Two vascular prostheses were used for aortic replacement (Hemashield Gold, $34 \mathrm{~mm}$ and $28 \mathrm{~mm}$ ). The innominate and left carotid arteries were attached to the ascending aortic prosthesis using a separate vascular prosthesis (Hemashield Gold, $20 \mathrm{~mm}$ ). After prolonged postoperative bleeding with rethoracotomy on the first postoperative day, the patient's condition improved until postoperative day 6 , when a sudden and severe elevation of transaminase levels was detected (glutamic oxaloacetic transaminase: 10,900 U/L; glutamic pyruvic transaminase: $6200 \mathrm{U} / \mathrm{L}$ ). A new CT scan was obtained that showed an obstruction of the true aortic lumen by the false lumen, and the patient was taken to the operating room.

After the antegrade implantation of a Gore TAG stent, the perfusion of the true lumen was improved (Figure 1,C), and liver enzymes decreased to normal values during the next few days. The patient was discharged without remaining impairment.

\section{DISCUSSION}

The presented technique of open antegrade endovascular aortic repair is safe and easy to perform, even in an emergency setting. Interventional treatment of the dissected aorta after surgical treatment of Stanford type A dissection is a therapeutic option that has been described by various groups. The clinical indications for this type of procedure are still the subject of debate, with some centers even evaluating the routine use as a preventive measure, ${ }^{5}$ although there is still an ongoing debate regarding the long-term benefit of this procedure in patients with Stanford type A dissection, especially in regard to the long-term results with respect to the related data from the Endovascular Aneurysm Repair (EVAR) and Investigation of Stentgrafts in Aortic Dissection (INSTEAD) trials. ${ }^{6}$

Because of the lack of evidence, we do not use stent graft placement in a preventive manner. However, in the case of severe complications, it has proven to be a valuable tool.

The presented technique addresses a variety of complications, including malperfusion, a serious complication occurring in $30 \%$ of the patients with aortic type A dissection. ${ }^{7,8}$ Established treatment options include conventional transfemoral placement of a stent graft and fenestration of the intimal flap. ${ }^{9}$ Although conventional placement of a stent graft has the advantage of avoiding a resternotomy, our experience with the antegrade implantation is that the exact deployment of the stent can be achieved fast, and it is technically uncomplicated because of direct implantation into the true lumen through the implanted prosthesis. Because of the short distance to the landing zone, there is less potential for injury to the friable dissected aorta even in cases with complicated anatomy. Furthermore, there is the possibility to surgically attach the stent to the vascular prosthesis. The stent can be deployed independently of the diameter or the condition of the infra-aortic vessels. Although transcutaneous implantation is the less-invasive choice, the risk for sternal infection or mediastinitis is low in the hands of an experienced surgeon. Nevertheless, in case of additional risk factors for wound infection (eg, obesity and diabetes), we would omit this approach because of the elevated risk of mediastinitis.

Decompression of the false lumen with fenestration of the intimal flap can be used to treat malperfusion syndrome, but this is rarely performed as a stand-alone treatment. The largest published study involving 40 patients by Slonim and colleagues ${ }^{10}$ indicated that although reperfusion could be achieved in $93 \%$ of all cases, a complex intervention including an additional aortic stent graft usually was required in the majority of the patients (in this study, only 2 of the patients received fenestration alone). ${ }^{11}$

Our established clinical approach in cases of malperfusion in aortic dissection is therefore the primary attempt to achieve reperfusion by means of aortic stent placement via retrograde or antegrade implantation; additional measures, such as fenestration, are considered if stent placement alone is insufficient or in the case of a complex pathology.

By using the described procedure, a situation similar to the frozen elephant trunk (FET) procedure can be achieved. Our technique may be used instead of an FET, especially if the necessity of an FET is not clear during the primary operation. Antegrade stent implantation does not interfere with the primary technique for aortic repair; furthermore, the times of cardiocirculatory arrest and extracorporeal circulation are reduced in comparison with FET. The FET procedure still may be advantageous in certain situations, especially in cases of a difficult distal anastomosis after arch replacement because of fragile tissue.

\section{CONCLUSIONS}

According to our still limited experience, this technique is safe to perform. Our technique can be deployed after decannulation or as a separate treatment option without the need for an initial decision about the treatment of the descending aorta, thus offering greater flexibility in the therapeutic approach. In individual cases, this technique could be used as an alternative to the FET procedure. 


\section{References}

1. Follis F, Filippone G, Stabile A, et al. Endovascular graft deployment in the false lumen of type B dissection. Interact Cardiovasc Thorac Surg. 2010;10: $597-9$.

2. Mestres CA, Fernandez C, Josa M, et al. Hybrid antegrade repair of the arch and descending thoracic aorta with a new integrated stent-Dacron graft in acute type A aortic dissection: a look into the future with new devices. Interact Cardiovasc Thorac Surg. 2007;6:257-9.

3. Roselli EE, Soltesz EG, Mastracci T, et al. Antegrade delivery of stent grafts to treat complex thoracic aortic disease. Ann Thorac Surg. 2010;90: 539-46.

4. Probst C, Esmaizadeh B, Schiller W, Wilhelm K. Emergent antegrade endovascular stent placement in a patient with perforated Stanford B dissection via right axillary artery. Eur J Cardiothorac Surg. 2008;33:1148-9.

5. Uchida N, Ishihara H, Shibamura H, Kyo Y, Ozawa M. Midterm results of extensive primary repair of the thoracic aorta by means of total arch replacement with open stent graft placement for an acute type A aortic dissection. $J$ Thorac Cardiovasc Surg. 2006;131:862-7.
6. Dobrilovic N, Elefteriades JA. Stenting the descending aorta during repair of type A dissection: technology looking for an application? J Thorac Cardiovasc Surg. 2006; 131:777-8.

7. Cambria RP, Brewster DC, Gertler J, et al. Vascular complications associated with spontaneous aortic dissection. J Vasc Surg. 1988;7:199-209.

8. Trimarchi S, Nienaber CA, Rampoldi V, et al. Contemporary results of surgery in acute type A aortic dissection: The International Registry of Acute Aortic Dissection experience. J Thorac Cardiovasc Surg. 2005;129:112-22.

9. Midulla M, Fattori R, Beregi JP, Dake M, Rousseau H. Aortic dissection and malperfusion syndrome: a when, what and how-to guide. Radiol Med. 2012 Apr 1 [Epub ahead of print].

10. Slonim SM, Miller DC, Mitchell RS, Semba CP, Razavi MK, Dake MD. Percutaneous balloon fenestration and stenting for life-threatening ischemic complications in patients with acute aortic dissection. J Thorac Cardiovasc Surg. 1999; 117:1118-26.

11. Midulla M, Renaud A, Martinelli T, et al. Endovascular fenestration in aortic dissection with acute malperfusion syndrome: immediate and late follow-up. J Thorac Cardiovasc Surg. 2011;142:66-72. 